\title{
Práticas alimentares e estado nutricional de crianças no segundo semestre de vida atendidas na rede pública de saúde
}

\author{
Feeding practices and nutritional status of \\ children in the second semester of life who \\ receive care in public health facilities
}

Simone Paula MODESTO ${ }^{1,2}$

Macarena Urrestarazu DEVINCENZI ${ }^{2}$

Dirce Maria SIGULEM ${ }^{3}$

\section{R E S U M O}

\section{Objetivo}

Avaliar o estado nutricional e as práticas alimentares de crianças no segundo semestre de vida atendidas na rede pública de saúde do município de Taboão da Serra, SP.

\section{Métodos}

Estudo de corte transversal conduzido em Unidades Básicas de Saúde, classificadas em dois agrupamentos (região central e região periférica). A amostra foi constituída por 180 crianças, sendo 90 de cada agrupamento. O consumo alimentar foi registrado pelo método recordatório de 24 horas. Foram tomadas medidas de peso e dosagem de hemoglobina por punção capilar.

\section{Resultados}

A prevalência de anemia encontrada foi de $30,5 \%$ sem diferença entre os grupos. Os valores das medianas do escore- $Z$ (peso/idade) foram: 0,02 e 0,03, para os agrupamentos central e periférico, respectivamente. A utilização de suplemento de ferro apresentou diferença entre os grupos $(p=0,001)$, sendo mais presente no agrupamento periférico, enquanto que para a vitamina $A$, a suplementação foi significantemente maior no agrupamento central $(p=0,044)$. Verificou-se introdução precoce de alimentos distintos do leite materno na dieta infantil e diferença significante entre os grupos na idade de introdução de chá, suco de fruta, sopa e sopa com carne no esquema alimentar. Em relação à ingestão de nutrientes verificou-se consumo adequado de energia e proteínas, mas quanto aos micronutrientes estudados, há grande probabilidade de inadequação do consumo de ferro e vitamina $\mathrm{C}$.

\footnotetext{
1 Prefeitura Municipal de Taboão da Serra. Estrada Tenente José Maria da Cunha, 862, Jd. Record, 06783-230, Taboão da Serra, SP, Brasil. Correspondência para/Correspondence to: S.P. MODESTO. E-mail: <simodesto@terra.com.br>

2 Universidade Federal de São Paulo, Departamento de Ciências da Saúde. Santos, SP, Brasil.

3 Universidade Federal de São Paulo, Escola Paulista de Medicina, Programa de Pós-Graduação em Nutrição. São Paulo, SP, Brasil.
} 


\section{Conclusão}

A prevalência de anemia é relativamente reduzida, comparada com os dados obtidos em outros locais, e o esquema de introdução de alimentos complementares é inadequado, frente às recomendações atuais. A utilização de suplementos de nutrientes deve ser revista.

Termos de indexação: anemia ferropriva; desmame; estado nutricional; lactente.

\section{A B S T R A C T}

\section{Objective}

To assess the nutritional status and feeding practices of children in the second semester of life who receive care in public health facilities of the city of Taboão da Serra, SP.

\section{Methods}

This is a cross-sectional study done in basic healthcare units divided into two groups: downtown and suburb. The sample was comprised of 180 children, 90 in each group. Food consumption was determined by the 24-hour recall. Weight and hemoglobin (by the capillary puncture method) were recorded.

\section{Results}

The prevalence of anemia was $30.5 \%$ with no difference between the groups. The median scores- $Z$ (weight-for-age) were: 0.02 and 0.03 , for the downtown and suburb groups, respectively. The use of iron supplements was different between the groups ( $p=0.001)$ : the suburb group used it more often. Meanwhile, vitamin A supplementation was significantly higher in the downtown group $(p=0.044)$. Foods other than breast milk were introduced early. There was a significant difference between the groups regarding the age when other foods were introduced, such as tea, juice, soup and soup with meat. In relation to nutrient intake, energy and protein intake were adequate but among the studied micronutrients, intake of vitamin $\mathrm{C}$ and iron were likely to be inadequate.

\section{Conclusion}

The prevalence of anemia is relatively low when compared with data obtained from other places and the weaning diet is inadequate when compared with the current recommendations. The use of nutrient supplements should be reviewed.

Indexing terms: anemia, iron deficiency; weaning; nutritional status; infant.

\section{N T R O D U Ç Ã O}

Durante os dois primeiros anos de vida a criança apresenta acelerado crescimento e desenvolvimento, com expressivas aquisições psicomotoras e neurológicas. A desnutrição e as infecções são particularmente comuns nesse período, especialmente nos países em desenvolvimento, e há relevante prevalência de carências nutricionais nesse estágio de vida. Dada a vulnerabilidade desse período, os cuidados em saúde destinados à criança menor de dois anos devem enfatizar a prática alimentar adequada, que será importante não só para prevenir ou minimizar deficiências nutricionais, mas também para não prejudicar a saúde da criança a curto prazo (reduzindo a morbi-mortalidade infantil), nem deixar seqüelas futuras como retardo de crescimento, atraso escolar e desenvolvimento de doenças crônicas ${ }^{1-3}$.

Nesse contexto, o segundo semestre de vida pode ser considerado o período mais crítico, pois nessa idade as necessidades nutricionais da criança aumentam, principalmente, as demandas de energia e nutrientes específicos (ferro, por exemplo), e o leite materno sozinho não tem mais condições de suprir as necessidades nutricionais ${ }^{3}$. Nessa fase os alimentos complementares são incluídos na dieta e a criança irá se adaptar ao padrão alimentar da família e da comunidade onde vive.

A desnutrição tem sido responsável, direta ou indiretamente, por $60 \%$ das 10,9 milhões de 
mortes de crianças menores de cinco anos de idade. Aproximadamente dois terços dessas mortes, que, freqüentemente, são associadas com práticas alimentares inadequadas, ocorrem no primeiro ano de vida ${ }^{4}$.

Quanto à deficiência de ferro, ela pode afetar o desenvolvimento cognitivo e o crescimento físico das crianças, assim como a imunidade e a morbidade por infecções, sendo que as maiores prevalências ocorrem no segundo ano de vida ${ }^{5}$. Por esse motivo é que a adequação dietética dos micronutrientes, principalmente em relação ao ferro, deve ser uma preocupação constante no período de alimentação complementar, pois o risco de anemia aumenta ao longo do primeiro ano de vida: de 33,7 para $71,8 \%$ do primeiro para o segundo semestre de vida, conforme dados da cidade de São Paulo, em 1996 .

Diante do que foi exposto, a amamentação é extremamente importante para a criança, pois o leite materno, além de ser um alimento completo, adequado para a criança, com boa disponibilidade de nutrientes (inclusive de ferro), possui fatores protetores contra infecções. Deve ser oferecido de maneira exclusiva, nos primeiros seis meses de vida da criança, e mantido até os dois anos de idade ou mais ${ }^{1}$.

Conforme definição da Organização Mundial da Saúde (OMS), o período de alimentação complementar é aquele durante o qual outros alimentos ou líquidos são oferecidos à criança junto com o leite materno. Qualquer alimento ou líquido que contenha nutrientes, oferecido à criança nesse período, é chamado de alimento complementar.

A alimentação complementar adequada deve compreender alimentos ricos em energia, proteínas e micronutrientes (com destaque para ferro, zinco, cálcio, vitamina $\mathrm{A}$, vitamina $\mathrm{C}$ e ácido fólico), livres de contaminação (biológica, química ou física), de fácil consumo e aceitação pela criança, com custo aceitável e preparados a partir dos alimentos da famíliå.

Na literatura existem poucos trabalhos que abordam as práticas alimentares do lactente e, normalmente, os artigos científicos descrevem o perfil de introdução de alimentos, tipo de aleitamento materno praticado, sem quantificar o consumo alimentar9-11.

Dada a relevância da alimentação infantil, este estudo buscou avaliar as práticas alimentares, quantificando o consumo alimentar de crianças no segundo semestre de vida, atendidas na rede básica de saúde do município de Taboão da Serra, SP.

\section{M É T O D O S}

Foi conduzido um estudo de corte transversal, em Unidades Básicas de Saúde (UBSs) de Taboão da Serra, região sudoeste da Grande São Paulo. O município apresenta área de $20,92 \mathrm{~km}^{2}$ e 220.515 habitantes na área urbana ${ }^{12}$.

As UBSs foram classificadas em dois agrupamentos: região central e região periférica, definidos conforme porcentagem de domicílios em área de habitação sub-normal (favelas) e instrução da população geral e do chefe de família ${ }^{13}$.

A coleta de dados foi realizada entre os meses de fevereiro e setembro de 2004. Para compor a amostra os indivíduos foram selecionados de acordo com os seguintes critérios de elegibilidade: ter idade entre 6 e 11 meses, residir em Taboão da Serra e freqüentar a UBS para acompanhamento do crescimento e do desenvolvimento. Foram excluídos da amostra os gemelares e crianças nascidas com peso inferior a $2500 \mathrm{~g}$.

A seleção para compor a amostra ocorreu conforme demanda espontânea, no momento da consulta para acompanhamento de desenvolvimento e crescimento e/ou vacinação. A amostra envolve 180 crianças, sendo 90 integrantes em cada agrupamento. Quanto à faixa etária, cada agrupamento é constituído por 45 crianças com idade entre 6 e 8 meses e 45 crianças com idade entre 9 e 11 meses.

As crianças somente foram incluídas no estudo após leitura e assinatura, pelos responsáveis, do termo de consentimento livre e esclarecido. Este trabalho foi aprovado pelo Comitê 
de Ética em Pesquisa da Universidade Federal de São Paulo/Hospital São Paulo.

Foi adotado um questionário que incluía informações sobre a criança, sobre os pais, moradia e histórico da introdução de alimentos. O consumo alimentar foi registrado pelo método recordatório de 24 horas. As mães (ou responsáveis) foram questionados a respeito dos alimentos e preparações consumidas pela criança (e respectivas porções em medidas caseiras), fornecendo aos entrevistadores informações sobre a alimentação da criança na época da entrevista. Além disso, a entrevista abordou também a idade da criança em que determinados alimentos foram introduzidos na dieta infantil, buscando o detalhamento sobre a época da vida da criança em que a mãe iniciou o oferecimento de água, chá, frutas e alimentos fonte de ferro. Para auxiliar a determinação do tamanho das porções foram utilizados utensílios domésticos (colheres, conchas, mamadeiras) e desenhos demonstrativos de porções de carnes (bifes, cubos, pedaços). Os cálculos foram elaborados com a utilização do software Sistema de apoio à Decisão em Nutrição, versão $2.5^{14}$.

Os consumos de energia, proteína, ferro, vitaminas A e C, assim como a densidade de ferro da dieta, foram comparados considerando-se os dois agrupamentos. Foi aferido, também, o número de crianças que apresentaram consumo de energia e proteína inferior às recomendações atuais. Para energia e densidade de ferro as recomendações da OMS foram estabelecidas como parâmetro ${ }^{2,7}$. Para os demais nutrientes estudados, considerou-se a Recommended Dietary Allowance (RDA), Estimated Average Requirement (EAR) ou Adequate Intake (AI) referentes ao segundo semestre de vida. O consumo de proteína foi comparado com o valor preconizado pela RDA $(1,2 \mathrm{~g}$ por quilo de peso corporal/dia) e, para avaliar o consumo de ferro, utilizou-se o valor registrado pela $\operatorname{EAR}(6,9 \mathrm{mg}$ por dia). Em relação às vitaminas A e $C$ foram consideradas as Als $50 \mu \mathrm{g}$ retinol equivalente e 50mg por dia, respectivamente ${ }^{15-18}$.
A utilização de suplemento de ferro e vitamina A também foi comparada considerando-se os dois agrupamentos.

As medidas de peso foram tomadas com a criança despida, utilizando balança eletrônica, de plataforma, marca Kratos (capacidade máxima de $150 \mathrm{~kg}$ e precisão de $50 \mathrm{~g}$ com tara) instalada em superfície plana. Foram calculados o valor mediano e os percentis 25 e 75 do indicador peso para idade, expresso em unidades de desvio-padrão (escore-Z) para caracterizar o estado nutricional da criança. O cálculo do escore-Z foi realizado com o programa Epi Info versão 6.0, que utiliza como referência a curva do National Center for Health Statistics de 1977 (NCHS) ${ }^{19}$. Adotou-se como ponto de corte o escore- $Z \leq-2$ para definir déficit nutricional, segundo a $\mathrm{OMS}^{20}$.

A dosagem de hemoglobina foi feita com o hemoglobinômetro portátil marca HemoCue. A coleta de sangue foi realizada por punctura do calcanhar, com uso de lancetas descartáveis e o resultado da medida comunicado imediatamente ao responsável. Todas as aferições dos níveis de hemoglobina foram registradas nos prontuários das crianças. Nas situações em que foi diagnosticada anemia, os responsáveis foram informados quanto à terapia nutricional e as crianças foram encaminhadas ao pediatra para acompanhamento. Conforme recomendação da OMS, foi considerado ponto de corte para anemia o valor de $11 \mathrm{~g} / \mathrm{dL}_{\mathrm{dL}}$ de hemoglobina ${ }^{5}$.

Nas UBSs não há atendimento de nutrição, as crianças são acompanhadas por pediatras e equipe de enfermagem. Após o término da coleta de dados, todas as mães ou responsáveis receberam orientação nutricional específica de acordo com o estágio de vida de cada criança.

Para tabulação dos dados e análise estatística, foram utilizados os programas Epi Info 6.0 e SigmaStat ${ }^{19,21}$.

Nas tabelas que reúnem os resultados da pesquisa, o número de crianças em cada agrupamento é sempre 90, com exceção da variável duração da amamentação. Os valores de $p$ significantes estão indicados por asterisco (*). 
Para análise dos resultados foram aplicados os testes: qui quadrado, " $t$ " de Student e Mann-Whitney. Foi considerado, como significância estatística, o valor de $p \leq 0,05$.

\section{RESULTADOSE DISCUSSÃO}

Neste trabalho foram analisadas variáveis que podem influenciar o estado nutricional e a prevalência de deficiência de ferro como: idade, sexo, estados fisiológico e patológico, além de condições ambientais e socioeconômicas ${ }^{5,22}$.

Na população integrante da análise, não foi encontrada diferença estatística entre os resultados relativos ao sexo considerando-se os dois agrupamentos. Em relação à variável peso ao nascer, o agrupamento central apresentou valor médio de $3316 \mathrm{~g}$ (desvio-padrão - $\mathrm{DP}=407,5)$ e o agrupamento periférico $3274,5 \mathrm{~g}$ ( $D P=407,3)$, sem diferença estatística entre os grupos $(p=0,472)$. É importante lembrar que as crianças com peso ao nascer inferior a 2500g não integraram a amostra da pesquisa.

Quanto às características maternas, os valores relativos às medianas de idade verificados nos agrupamentos central e periférico foram 25 e 24 anos, respectivamente, sem diferença estatística entre os grupos $(p=0,478)$. A escolaridade da mãe verificada no agrupamento central (mediana de 9,5 anos de estudo) é estatisticamente superior $(p=0,018)$ à escolaridade das mães do agrupamento periférico (mediana de 8 anos de estudo), demonstrando que os critérios de separação das UBSs em agrupamentos são coerentes com os resultados encontrados.

Na entrevista, foram abordadas, também, questões relativas a condições familiares e ambientais, como número de irmãos, ocorrência de internação hospitalar, ocupação paterna e materna, número de cômodos na residência, condições estas que demonstraram homogeneidade entre os grupos. A única característica ambiental que mostrou diferença importante foi o número de domicílios atendidos por rede de esgoto sanitário $(p=0,0512)$, sendo mais presente no agrupamento central. Percebe-se, então, que as crianças do agrupamento periférico estão mais expostas a condições desfavoráveis (baixa escolaridade da mãe e saneamento básico precário), quando comparadas com as crianças do outro agrupamento.

A análise das práticas alimentares mostrou que as prevalências de amamentação são baixas, independentemente de agrupamento (Tabela 1). Entre as 108 crianças que já não consumiam leite materno, a duração mediana da amamentação

Tabela 1. Prevalência e duração (dias) da amamentação, tipo de leite consumido e ingestão de alimentos fonte de ferro, em dois agrupamentos de crianças* (central e periférico). Taboão da Serra, SP, 2004.

\begin{tabular}{|c|c|c|c|}
\hline \multirow{2}{*}{ Variável } & \multicolumn{2}{|c|}{ Agrupamento } & \multirow{2}{*}{$p$} \\
\hline & Central & Periférico & \\
\hline Prevalência amamentação ${ }^{\mathbf{a}}$ & $32(35,6 \%)$ & $40(44,4 \%)$ & 0,224 \\
\hline Duração amamentação (dias) $)^{\mathbf{b}, \mathbf{c}}$ & $90(60-150)$ & $98(45-180)$ & 0,275 \\
\hline \multicolumn{4}{|l|}{ Tipo de leite ${ }^{a}$} \\
\hline Materno & $19(21,1 \%)$ & $22(24,4 \%)$ & \multirow{3}{*}{0,445} \\
\hline Misto (materno+leite de vaca) & $13(14,4 \%)$ & $18(20,0 \%)$ & \\
\hline Leite de vaca & $58(64,5 \%)$ & $50(55,6 \%)$ & \\
\hline \multicolumn{4}{|l|}{ Fontes de ferro ${ }^{a}$} \\
\hline Sem feijão, sem carne & $16(17,8 \%)$ & $13(14,4 \%)$ & \multirow{4}{*}{0,760} \\
\hline Sem feijão, com carne & $15(16,7 \%)$ & $20(22,2 \%)$ & \\
\hline Com feijão, sem carne & $9(10,0 \%)$ & $10(11,1 \%)$ & \\
\hline Com feijão, com carne & $50(55,5 \%)$ & $47(52,3 \%)$ & \\
\hline
\end{tabular}

* Para as análises foram consideradas 180 observações válidas, ateste qui-quadrado, freqüências observadas; ' bn:108; 'cteste de Mann-Whitney, valores da mediana (25으 percentil e 75으 percentil entre parênteses). 
não atingiu os 4 meses de idade nos dois agrupamentos. É importante lembrar que não é possível definir a duração mediana da amamentação na amostra estudada, pois 72 crianças (40\%) ainda eram amamentadas no momento da entrevista. Embora estas crianças ainda sejam acompanhadas na unidade de saúde, estes dados revelam que o desmame precoce é prática comum nesta população. Portanto, fica evidente que políticas de promoção e apoio à amamentação devem ser implementadas no município.

Substancial parcela das crianças dos dois agrupamentos (78,9\% no central e $75,6 \%$ no periférico) faziam uso de leite de vaca (associado ou não ao leite materno). O consumo de fórmula infantil encontrado na época da entrevista foi mínimo, sendo de apenas 3 crianças no agrupamento central e 2 no periférico. Destaca-se que o consumo de leite de vaca por crianças menores de 12 meses tem sido extremamente discutido no meio científico. Os principais questionamentos são de que o leite de vaca é um alimento pobre em ferro, e este é de baixa biodisponibilidade, pode causar perda de sangue pelo trato gastrintestinal e pode levar à sobrecarga renal de solutos (pela elevada quantidade de proteínas e alguns minerais em sua composição). Assim, a Organização Mundial de Saúde registra que se o leite de vaca é parte importante da dieta, devem ser utilizados suplementos de ferro, alimentos fortificados de boa biodisponibilidade, além de propiciar adequada hidratação da criança, principalmente, na presença de diarréia, minimizando os possíveis danos causados pelo consumo desse tipo de leite ${ }^{2}$.

Quanto ao consumo de alimentos fonte de ferro, observou-se que, aproximadamente, metade das crianças consumia carne e feijão nos dois agrupamentos (Tabela 1). Devincenzi et al. ${ }^{23}$ identificaram, entre crianças de baixa renda com idade entre 6 e 12 meses, que o consumo de feijão, carne e alimentos fonte de vitamina $C$ é fator protetor para anemia aos 6 meses de idade, indicando que a alimentação adequada pode prevenir ou minimizar os efeitos da anemia ferropriva na infância.
Na comparação do perfil de introdução de alimentos, apresentado na Tabela 2, com os registros disponíveis na literatura, verifica-se a introdução precoce de alimentos distintos do leite materno ${ }^{1,3}$. Água, chá, fruta (papa e suco), sopa e sopa com carne são introduzidos antes dos 6 meses de idade. Os alimentos considerados fonte de ferro (feijão e carne) foram os últimos a serem incorporados à dieta, mas, ainda assim, em época adequada (180 dias de vida), para os dois agrupamentos de crianças (considerando a idade mediana). Embora o consumo de alimentos fonte de ferro tenha sido iniciado em idade apropriada, essas crianças receberam alimentação complementar antes dos 6 meses de idade. Provavelmente, os alimentos substituíram o aleitamento materno, cuja duração foi verificada como reduzida na população estudada, como o comentado anteriormente. Vale lembrar que este trabalho teve por base uma amostra de crianças que se encontravam no segundo semestre de vida, sendo assim, as questões relativas ao histórico de introdução de alimentos referem-se a um período recente da vida da criança.

A introdução precoce de alimentos sólidos também foi verificada em outros estudos. Nesses trabalhos, os primeiros alimentos a integrarem a alimentação infantil foram as frutas, os cereais e as hortaliças. Os alimentos fonte de ferro (carne e

Tabela 2. Idade mediana e média (dias) de introdução de alimentos em dois agrupamentos de crianças* (central e periférico). Taboão da Serra, SP, 2004.

\begin{tabular}{|c|c|c|c|c|c|}
\hline \multirow{3}{*}{ Alimento } & \multicolumn{4}{|c|}{ Agrupamento } & \multirow{3}{*}{$p$} \\
\hline & \multicolumn{2}{|c|}{ Central } & \multicolumn{2}{|c|}{ Periférico } & \\
\hline & M & $\bar{x}$ & M & $\bar{x}$ & \\
\hline Água ${ }^{a}$ & 75,0 & 80,0 & 90,0 & 88,82 & 0,293 \\
\hline Cháa & 30,0 & 47,8 & 60,0 & 67,9 & 0,022 * \\
\hline Fruta (papa) & 120,0 & 121,0 & 120,0 & 130,7 & 0,092 \\
\hline Suco de fruta ${ }^{a}$ & 120,0 & 117,6 & 120,0 & 129,6 & 0,050 * \\
\hline Sopa $a^{a}$ & 127,5 & 138,5 & 150,0 & 150,5 & $0,019 *$ \\
\hline Sopa com carne $e^{a}$ & 150,0 & 145,6 & 165,0 & 158,2 & 0,031 * \\
\hline Arroz $^{\mathrm{a}}$ & 180,0 & 190,5 & 180,0 & 185,8 & 0,869 \\
\hline Carne $^{a}$ & 180,0 & 166,1 & 180,0 & 173,7 & 0,395 \\
\hline Feijãoa & 180,0 & 188,3 & 180,0 & 185,9 & 0,951 \\
\hline
\end{tabular}


feijão) foram introduzidos mais tardiamente. Apenas um desses trabalhos identificou consumo precoce de carne (entre 3 e 6 meses) ${ }^{9-11}$.

Vale ressaltar que foi encontrada diferença estatisticamente significante entre os dois agrupamentos, no tocante à idade de introdução de chá, suco de fruta, sopa e sopa com carne. De maneira geral, verifica-se que no agrupamento central, os alimentos são introduzidos mais precocemente (Tabela 2), talvez pelo maior acesso das famílias a alimentos diferentes do leite materno.

Neste estudo, houve a preocupação de analisar o valor nutricional das dietas a partir de uma análise quantitativa da alimentação, em relação a energia e alguns nutrientes críticos como proteínas, ferro e vitaminas A e C (Tabela 3). Não foi observada diferença estatisticamente significante quanto à ingestão de nutrientes entre os dois agrupamentos.

Frente às recomendações atuais, poucas crianças apresentaram consumo de energia e proteínas inferior à ingestão recomendada (7,8\% e $8,3 \%$ respectivamente) $)^{2,15}$. Os valores medianos do consumo de ferro encontrados nos dois agrupamentos indicam que há grande probabilidade de inadequação do consumo deste nutriente uma vez que a ingestão mediana revelou-se inferior ao valor de EAR para esse grupo etário ${ }^{16}$.
Em relação às vitaminas $A$ e $C$, vale ressaltar que não há valores de RDA e EAR estabelecidos, sendo assim foram utilizados os valores de Al para avaliar o consumo dessas vitaminas. Em função das características desse parâmetro, não foi possível concluir com precisão desejável se o consumo desses nutrientes está adequado ou não. Os valores medianos de ingestão de vitamina $\mathrm{A}$ revelaram-se acima do valor de $\mathrm{Al}$ nos dois agrupamentos, enquanto que o consumo mediano de vitamina $\mathrm{C}$ revelou-se abaixo do valor de $\mathrm{Al}$ nos dois agrupamentos (Tabela 3).

Estes resultados indicam algumas prováveis deficiências dietéticas, sendo que se faz necessário melhorar a qualidade da alimentação infantil, com a inclusão de alimentos selecionados considerados fonte de nutrientes, além de revisar a rotina de suplementação dessas crianças.

Em relação à vitamina $C$, trata-se de um nutriente cujas fontes são de fácil acesso e têm boa aceitação pela criança ${ }^{17}$. Além disso, a vitamina $C$ é de reconhecida importância para otimizar a absorção de ferro não heme. Da mesma maneira, os alimentos fonte de vitamina A podem ser facilmente incorporados à alimentação infantil, destacando-se a importância da orientação nutricional.

Tabela 3. Ingestão mediana de energia, proteínas, vitamina C, ferro, zinco, vitamina A e densidade de ferro do alimento complementar segundo agrupamento de crianças* (central e periférico). Taboão da Serra, SP 2004.

\begin{tabular}{lcc}
\hline \multirow{2}{*}{ Variável } & Agrupamento & \\
\cline { 2 - 3 } & Central & Periférico \\
\hline
\end{tabular}

\begin{tabular}{|c|c|c|c|c|c|}
\hline \multirow{2}{*}{\multicolumn{6}{|c|}{ Energia (kcal/dia) a }} \\
\hline & & & & & \\
\hline 6 a 8 meses de idade & 984,8 & $(760,9-1136,2)$ & 883,8 & $(716,6-988,8)$ & 0,135 \\
\hline 9 a 12 meses de idade & 1006,4 & $(788,9-1148,7)$ & 986,3 & $(819,8-1236,5)$ & 0,790 \\
\hline Proteínas (g/kg/dia)a & 3,5 & $(2,5-4,8)$ & 3,2 & $(2,1-4,1)$ & 0,151 \\
\hline Vitamina C (mg/dia) ${ }^{a}$ & 43,6 & $(28,2-87,6)$ & 47,3 & $(31,2-81,8)$ & 0,729 \\
\hline Ferro $(\mathrm{mg} / \mathrm{dia})^{\mathrm{a}}$ & 4,1 & $(2,1-7,2)$ & 3,6 & $(2,2-5,5)$ & 0,193 \\
\hline Vitamina A ( $\mu \mathrm{g} / \mathrm{dia})^{\mathbf{a}}$ & 879,2 & $(422,4-1811,7)$ & 1076,2 & $(425,6-2108,0)$ & 0,296 \\
\hline \multicolumn{6}{|l|}{ Densidade de Ferro ${ }^{\mathbf{a}}$} \\
\hline 6 a 8 meses de idade & 0,32 & $(0,17-0,60)$ & 0,36 & $(0,23-0,51)$ & 0,936 \\
\hline 9 a 12 meses de idade & 0,48 & $(0,32-0,64)$ & 0,38 & $(0,31-0,53)$ & 0,171 \\
\hline
\end{tabular}


O consumo de alimentos fonte de ferro não foi suficiente para garantir a adequada ingestão do mineral (Tabela 3), provavelmente porque nesse estágio de vida, a dieta inclui alimentos que são pobres em ferro, além de a quantidade de alimentos ingerida ser pequena. Resultados similares foram encontrados, no município de São Paulo, onde foi identificado consumo mediano de $3,6 \mathrm{mg}$ de ferro por dia para crianças com idade similar ${ }^{24}$. Apesar da inadequada ingestão de ferro, a maioria das crianças não apresentou alterações nos níveis de hemoglobina que indicassem anemia, como será discutido mais adiante. Entretanto, é provável que o consumo deficiente de ferro esteja comprometendo as reservas do mineral, aumentando o risco de anemia ferropriva no segundo ano de vida, uma vez que, nessa fase, a criança apresenta acelerado crescimento e as demandas de ferro estão ampliadas.

Recentemente, a densidade de ferro da dieta se revelou como um indicador mais eficaz que a ingestão diária do mineral, para identificar risco de anemia em crianças com idade entre 6 e 9 meses $^{25}$. Na Tabela 3, verifica-se que as dietas são inadequadas, considerando as recomendações da OMS de $4 \mathrm{mg}$ de ferro/100kcal dos 6 aos 8 meses e 2,4mg de ferro/100kcal dos 9 aos 12 meses $^{7}$. Novamente, verifica-se a importância da suplementação de ferro para corrigir a deficiência dietética do mineral.

Comparando-se o consumo mediano de ferro e de vitamina A, constata-se que a provável deficiência de ingestão das crianças seja muito mais preocupante no tocante ao primeiro do que à segunda. No entanto, como se observa na tabela 4 , a suplementação de vitamina A é muito mais presente que a de ferro, considerando os dois agrupamentos de crianças. Esse ponto merece destaque, pois as medidas preventivas de suplementação dependem do envolvimento efetivo das mães (ou responsáveis), para garantir a adesão ao tratamento. Uma vez que esse comprometimento existe em relação à suplementação de vitamina $A$, vale insistir na orientação das mães quanto à importância do ferro para a nutrição da criança, na tentativa de minimizar as desistências do tratamento, a despeito de intercorrências, como os efeitos colaterais da administração do mineral.

Quanto ao estado nutricional das crianças integrantes da amostra, os valores das medianas dos escores- $Z$ de peso para idade indicam eutrofia dos dois grupos (Tabela 4). Foram identificadas apenas 8 crianças com déficit (escore- $Z \leq-2$ ), sendo $6(6,7 \%)$ no agrupamento central e $2(2,2 \%)$ no agrupamento periférico $(p=0,139)$. Dados do município de São Paulo (1995/96) também revelaram baixas prevalências de déficit nutricional, indicando que a desnutrição infantil deixa de ser endêmica, tornando-se relativamente rara, mesmo em populações mais pobres. Esse declínio da desnutrição infantil é justificado por mudanças positivas que ocorreram no período quanto ao poder aquisitivo das famílias e do nível de escolaridade das mães. Também vale ressaltar que a melhoria dos antecedentes reprodutivos (ordem de nascimento e intervalo interpartal) e do acesso da população a serviços de saneamento e de saúde também contribuem, expressivamente, para o controle da desnutrição 22 .

Tabela 4. Escore-Z de peso/idade, prevalência de anemia e suplementação (ferro e vitamina A) nos agrupamentos de crianças* (central e periférico). Taboão da Serra, SP, 2004.

\begin{tabular}{|c|c|c|c|c|c|}
\hline \multirow{3}{*}{$\begin{array}{l}\text { Variável } \\
\text { Índice peso/idade }\end{array}$} & \multicolumn{4}{|c|}{ Agrupamento } & \multirow{3}{*}{$\frac{p}{0,894}$} \\
\hline & \multicolumn{2}{|c|}{ Central } & \multicolumn{2}{|c|}{ Periférico } & \\
\hline & 0,02 & $(-0,66 / 1,00)$ & 0,03 & $(-0,51 / 0,80)$ & \\
\hline Prevalência de anemia ${ }^{\mathbf{b}}$ & 27,00 & $(30,0 \%)$ & 28 & $(31,1 \%)$ & 0,871 \\
\hline Suplementação de ferrob & 31,00 & $(34,4 \%)$ & 53 & $(58,9 \%)$ & 0,001 * \\
\hline Suplementação de vitamina $A^{\mathbf{b}}$ & 83,00 & $(92,2 \%)$ & 74 & $(82,2 \%)$ & $0,044^{*}$ \\
\hline
\end{tabular}

* Para as análises foram consideradas 180 observações válidas; ${ }^{a}$ teste de Mann-Whitney, valores da mediana (25ำ percentil e 75으 percentil entre

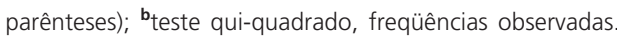


Em relação à prevalência de anemia, a amostra apresentou 30,6\% de crianças anêmicas sem diferença entre os grupos (Tabela 4), sendo que o valor médio dos níveis de hemoglobina foi de $11,63 \mathrm{~g} / \mathrm{dL}(\mathrm{DP}=1,36)$ no agrupamento central e $11,51 \mathrm{~g} / \mathrm{dL}(\mathrm{DP}=1,51)$ no agrupamento periférico. Das 55 crianças anêmicas, 11 (20,0\%) apresentaram anemia classificada como mais grave com níveis de hemoglobina inferiores a 9,5g/dL ${ }^{26}$, sendo 4 no agrupamento central ( $14,8 \%$ dos anêmicos deste grupo) e 7 no agrupamento periférico (25,0\% dos anêmicos do grupo).

As prevalências de anemia verificadas estão aquém de valores encontrados em outros locais, mas, considerando que essas crianças estão em acompanhamento na rede básica de saúde do município, o valor de 30,6\% de crianças anêmicas pode ser considerado expressivo. Em Embu, município vizinho de Taboão da Serra, foram identificados $74,1 \%$ de crianças anêmicas no grupo composto pelos menores de um ano ${ }^{27}$. No município de São Paulo, Sigulem et al. ${ }^{28}$ encontraram $41,3 \%$ de crianças anêmicas com idade entre 6 e 12 meses em 1974/1975. Ainda em São Paulo, em 1995/96 foram observados 71,8\% de crianças anêmicas com idade similar ${ }^{6}$. O que pode explicar essa diferença é que, neste trabalho, o grupo que compõe a amostra é exclusivamente usuário do serviço de saúde, portanto não representa a população. A representatividade populacional foi assegurada pelos autores dos artigos adotados para o estabelecimento das comparações. Em outro estudo similar a este, realizado em unidades de saúde da região central de São Paulo, também foram encontradas prevalências de anemia mais baixas (22,6\%) em crianças com idade entre 6 e 12 meses $^{29}$. Aparentemente, nas situações em que os estudos são conduzidos, tendo como base amostra composta por indivíduos atendidos em unidades de saúde, o seguimento de puericultura pode favorecer a condição de saúde das crianças, contribuindo com as menores prevalências de anemia identificadas.

Uma das medidas de prevenção da anemia em crianças menores de 2 anos, recomendada atualmente, é a suplementação medicamentosa de ferro ${ }^{5}$. Apesar disso, observou-se reduzida utilização do suplemento na população estudada (apenas $44,2 \%$ das crianças), sendo que a utilização foi significantemente maior no agrupamento periférico, como se verifica na tabela 4. Pode ser que a diferença decorra de uma maior preocupação da equipe de saúde, neste agrupamento, com a criança das regiões periféricas do município, mesmo em dissonância com as diretrizes atuais, que recomendam a suplementação universal. Ou ainda, que tal diferença se deva à decisão da mãe, que, supondo que a dieta oferecida à criança seja deficiente, opte por administrar o suplemento.

Quanto ao uso de suplemento de vitamina A, verificou-se comportamento inverso daquele observado e descrito para o ferro: a suplementação foi significantemente maior no agrupamento central (Tabela 4). Um dado que pode explicar esta diferença é o tipo de suplemento utilizado (ou prescrito). Apenas 9 crianças no agrupamento central $(10 \%)$ recebem suplementação de vitamina A por meio do polivitamínico distribuído na rede de saúde, e no periférico 18 crianças (20\%) o utilizam. É possível que, por inconstância no fornecimento à rede, estejam sendo prescritos outros suplementos que a família tenha que adquirir com recursos próprios, fator este que, no agrupamento periférico, pode ser limitante de sua utilização. A Pan American Health Organization sugere a suplementação medicamentosa em áreas endêmicas para deficiência de vitamina A, com administração de megadoses da vitamina a intervalos de 4 a 6 meses ${ }^{30}$. No acompanhamento ambulatorial de lactentes é conduta pediátrica freqüente, a suplementação universal até o final do primeiro ano de vida ${ }^{31}$.

Embora tenham sido encontradas diferenças entre os grupos quanto ao uso do suplemento, isso não interferiu na prevalência de anemia nem no estado nutricional das crianças. Ainda assim, estes dados são importantes, pois indicam que a rotina adotada, no tocante à suplementação, deve ser revista na rede de saúde.

Diante dos resultados discutidos neste trabalho, ressalta-se a importância da atuação do 
nutricionista na Unidade Básica de Saúde, uma vez que este profissional tem competência para elaborar o esquema de introdução de alimentos complementares, além de, juntamente com a equipe de saúde, orientar as mães (ou responsáveis) quanto à necessidade de suplementação. Ademais, é fundamental considerar que a introdução da alimentação complementar de maneira adequada pode ser decisiva para a saúde da criança nesse período vulnerável, além de permitir crescimento e desenvolvimento satisfatórios.

\section{REFERÊ NCIAS}

1. Brasil. Ministério da Saúde. Dez passos para uma alimentação saudável: guia alimentar para crianças menores de 2 anos. Brasília: Ministério da Saúde; 2002.

2. Dewey KG, Cohen RJ, Rollins NC. WHO technical background paper: feeding of nonbreastfed children from 6 to 24 months of age in developing countries. Food Nutr Bull. 2004; 25(4):377-402.

3. World Health Organization. Guiding principles for complementary feeding for the breastfed child. Geneva: PAHO; 2003.

4. World Health Organization. Global strategy for infant and young child feeding. Geneva: UNICEF; 2003.

5. World Health Organization. Iron deficiency anaemia assessment, prevention, and control: a guide for programme managers. Geneva; 2001.

6. Monteiro CA, Szarfarc SC, Mondini L. Tendência secular da anemia na infância na cidade de São Paulo (1984-1996). Rev Saúde Pública. 2000; 34(6 Suppl):62-72.

7. World Health Organization. Complementary feeding of young children in developing countries: a review of current scientific knowledge. Geneva; 1998.

8. World Health Organization. Complementary feeding: family foods for breastfed children. Geneva; 2000.

9. Marchioni DML, Latorre MRDO, Szarfarc SC, Souza SB. Complementary feeding: study on prevalence of food intake in two health centers of São Paulo city. Arch Latinoam Nutr. 2001; 51(2):161-6.

10. Souza SB, Szarfarc SC, Souza JMP. Prática alimentar no primeiro ano de vida, em crianças atendidas em centros de saúde escola do município de São Paulo. Rev Nutr. 1999; 12(2):167-74.
11. Tabai KC, Carvalho JF, Salay E. Aleitamento materno e prática de desmame em duas comunidades rurais de Piracicaba, SP. Rev Nutr. 1998; 11(2):173-83.

12. Fundação Sistema Estadual de Análise de Dados [citado em 16 Jun 2005]. Disponível em: http:// www.seade.gov.br

13. Taboão da Serra. Secretaria Municipal de Saúde. Plano de Saúde 2002-2005. Taboão da Serra: Prefeitura Municipal de Taboão da Serra; 2001.

14. Anção MS, Cuppari L, Tudisco LS, Draibe AS, Sigulem D. Sistema de apoio à decisão em nutrição-versão 2.5. São Paulo: Centro de Informática em Saúde da Universidade Federal de São Paulo/Escola Paulista de Medicina; 1993.

15. Dewey KG, Brown KH. Update on technical issues concerning complementary feeding of young children in developing coutries and implications for intervention programs. Food Nutr Bull. 2003; 24(1):5-28.

16. Institute of Medicine. Dietary reference intakes for vitamin A, vitamin K, arsenic, boron, chromium, copper, iodine, iron, manganese, molybdenum, nickel, silicon, vanadium, and zinc. Washington (DC): National Academy Press; 2001.

17. Institute of Medicine. Dietary reference intakes for vitamin C, vitamin E, selenium, and carotenoids. Washington (DC): National Academy Press; 2000.

18. Institute of Medicine. Dietary reference intakes for energy, carbohydrate, fiber, fat, fatty acids, cholesterol, protein, and amino acids (macronutrients). Washington (DC): National Academy Press; 2002.

19. Dean AG, et al. Epi Info [computer program]. Version 6: a word processing, database, and statistics program for epidemiology on micro-computers. Atlanta, Georgia: Centers of Disease Control and Prevention; 1994.

20. World Health Organization. Phisycal status: the use and interpretation of anthropometry. Geneva; 1995. Technical report series, 854.

21. SigmaStat for windows [computer program]. Version 2.0: a statiscal software. Jandel Corporation; 1995.

22. Monteiro CA, Conde WL. Tendência secular da desnutrição e da obesidade na infância na cidade de São Paulo (1974-1996). Rev Saúde Pública. 2000; 34(6 Supl):52-61.

23. Devincenzi MU, Colugnati FAB, Sigulem DM. Factores de protección para la anemia ferropriva: estúdio prospectivo em niños de bajo nível socioeconômico. Arch Latinoam Nutr. 2004; 54(2): 174-9.

24. Sichieri R, Szarfarc SC, Monteiro CA. Relação entre dieta e ocorrência de anemia ferropriva em crianças. J Pediatr. 1998; 64(5):169-74. 
25. Hadler MC, Colugnati FAB, Sigulem DM. Risks of anemia in infants according to dietary iron density and weight gain rate. Prev Med. 2004; 39(4): 713-21.

26. Monteiro CA, Szarfarc SC. Estudo das condições de saúde das crianças no município de São Paulo, SP (Brasil). 1984-1985: V - Anemia. Rev Saúde Pública. 1987; 21(3):255-60.

27. Terão SMI, Puccini RF, Silva EMK, Pedroso GC, Silva NN. Prevalência de anemia em crianças residentes no município de Embu (São Paulo), 1996-7. Rev Paul Pediatria. 2004; 22(1):7-14.

28. Sigulem DM, Tudisco ES, Goldenberg P, Athaide MMM, Vaisman E. Anemia ferropriva em crianças do município de São Paulo. Rev Saúde Pública. $1978 ; 12: 168-78$
29. Souza SB. Anemia no primeiro ano de vida em relação ao aleitamento materno. Rev Saúde Pública. 1997; 31(1):15-20.

30. Pan American Health Organization. Providing vitamin A supplements through immunization and other health contacts for children 6-59 months and women up 6 weeks postpartum: a guide for health workers; 2001 [cited 2005 Jul 10]; Available from: http:Ilwww.paho.org

31. Palma D. Alimentação no primeiro ano de vida. In: Lopez FA, Brasil ALD, organizadores. Nutrição e dietética em clínica pediátrica. São Paulo: Atheneu; 2003.

Recebido em: 9/9/2005

Versão final reapresentada em: 6/11/2006 Aprovado em: 15/6/2007 